\title{
A special Pencil of Binary Quartics.
}

\author{
By R. Vaidyayathaswamy.
}

(Received 25th January 1927. Read 4th March 1926.)

I. If the Jacobian sextic $a_{x}^{6}$ of a pencil of binary quartics is known, the pencil itself is determined in five ways. The explicit determination of the pencil in terms of the irrational invariants of $a_{x}{ }^{6}$ has been effected by Stephanos. ${ }^{1}$

There are two known cases in which a pencil of quartics admits of rational determination from its Jacobian. In these, the rationally determinable pencil differentiates itself algebraically from its remaining four co-Jacobian pencils.

The first of these cases is that of the syzygetic pencil. If $a_{x}{ }^{6}$ is the product of three mutually apolar quadratics $p_{x}{ }^{2} q_{x}{ }^{2} r_{x}{ }^{2}$, then it is known that there is a syzygetic pencil $\Gamma$ associated with it; the Jacobian of $\Gamma$ is $p_{x}{ }^{2} q_{x}{ }^{2} r_{x}{ }^{2}$, and every tetrad of $\Gamma$ is carried into itself by the three involutions determined by $p_{x}{ }^{2}, q_{x}{ }^{2}, r_{x}{ }^{2}$ respectively. It is easy to shew on the other hand that the four other co-Jacobian pencils $\Gamma_{1} \Gamma_{2} \Gamma_{3} \Gamma_{4}$ are all distinct from one another and from $\Gamma$, and are interchanged in pairs by the same three involutions. Thus the syzygetic pencil $\Gamma$ which is rationally determinable from $p_{s}{ }^{2} q_{c}{ }^{2} r_{s}{ }^{2}$ distinguishes itself algebraically from the remaining co-Jacobian pencils $\Gamma_{1} \Gamma_{2} \Gamma_{3} \Gamma_{4}$ in that it is transformed into itself by each of the three involutions.

The second case is that of the Null Pencil, studied in detail in a previous paper. ${ }^{2}$ When $a_{x}{ }^{6}$ is a perfect square $\left(b_{x}^{3}\right)^{2}$, then only one of the five co-Jacobian pencils which it determines is non-singular, and it distinguishes itself algebraically from the remaining four by this very fact. This non-singular pencil is the Null Pencil defined by the cubic $b_{x}{ }^{3}$.

In both these cases the result has apparently been brought about by the fact that the given Jacobian admits an automorphic linear group. In the former case in which the Jacobian is the product of three mutually apolar quadratics, the automorphic group

\footnotetext{
1 Mem. de linstitut, 27 (1883), 2.

2 The Null Pencil of Binary Quartics, Proc. Lond. Math. Soc., 2, 23 (1923) 317-325.
} 
is the octahedral group, isomorphic with the symmetric group on four symbols; in the latter case, it is the dihedral group isomorphic with the group on three symbols.

We shew here that there is a more general case of rational determination of the pencil from the roots of its Jacobian, in which the Jacobian does not admit an automorphic linear group. The theorem which will be proved runs as follows:

If the cubics $a_{x}{ }^{3}, a_{x}{ }^{3}$ are apolar, then we can determine a pencil $\Gamma$ rationally in terms of the coefficients of $a_{x}^{3}, a_{x}^{\prime}$, so as to have $a_{,}^{3} a_{x}^{\prime}{ }^{3}$ for its Jacobian; in other words, one of the five co-Jacobian pencils determined by a Jacobian sextic which is the product of two apolar cubics, is algebraically of a different character from the other four.

This theorem includes the Null Pencil as a particular case; for the Jacobian of the Null pencil is a squared cubic which is a particular case of the product of two apolar cubics. It is a consequence of a general theorem on linear systems of binary forms, which we shall first state and prove.

\section{A Theorem concerning Linear Systems of Binary Forms.}

II. By a "linear system $I_{n}{ }^{p}$ " we shall understand the totality of binary forms of the shape:

$$
\lambda_{1} f_{1}(x)+\lambda_{2} f_{2}(x)+\ldots+\lambda_{p} f_{p}(x)
$$

where $f_{1} f_{2} \ldots f_{p}$ are $p$ linearly independent binary $n$-ics. By the 'Jacobian' of $I_{n}{ }^{p}$ we shall mean the form of order $p(n+1-p)$ whose roots are $p$-ple roots of members of the system. By differentiating the general member of $I_{n}{ }^{p}(p-1)$ times and eliminating $\lambda_{1} \lambda_{2} \ldots \lambda_{p}$, it is clear that its Jacobian is the determinant:

where

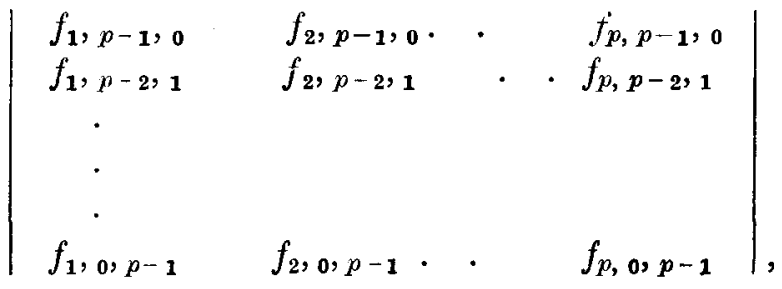

$$
f_{k, r, s}=\frac{d^{r+s} f_{k}}{d \alpha_{1}{ }^{r} d x_{2}{ }^{s}}
$$

The Jacobian is the fundamental combinant of the system, and is, it will be noticed, linear in the coefficients of each $f_{1}, f_{2}, \ldots f_{p}$. 
The theorem in question is as follows:

If an $I_{n}{ }^{p}$ varies in such wise as to always contain a fixed $I_{n}^{p-1}$ and to be always contained in a fixed $I_{n}{ }^{p+1}$, its Jacobian $J_{p(n+1-p)}$ will generate a pencil $\left[J_{p(n+1-p)}\right]$; the Jacobian of this pencil is simply the product of the Jacobians of the $I_{n}{ }^{p-1}$ and the $I_{n}{ }^{p+1}$.

It is clear first of all that there are $\infty^{\prime} I_{n}{ }^{p}$ 's satisfying the given conditions, and that one such given $I_{n}{ }^{p}$ can be found to contain any general member of the $I_{n}{ }^{p+1}$. From the linearity of the Jacobian it is also clear that the Jacobians of the variable $I_{n}{ }^{p}$ must generate a pencil $\left[J_{p(n-p+1)}\right]$.

To prove the second part, let $\beta$ be a root of the Jacobian of the $I_{n}{ }^{p+1}$, so that $I_{n}{ }^{p+1}$ contains a member of the form $(\beta x)^{p+1} \phi(x)$; the particular $I_{n}{ }^{p}$ which contains this member must consequently have $\beta$ for a double root of its Jacobian. $\beta$ being thus a double root of a $J_{p(n-p+1)}$, must be a root of the Jacobian of the pencil $\left[J_{p(n-p+1)}\right]$. Thus the Jacobian of the pencil contains the Jacobian of the $I_{n}^{p+1}$ as a factor. Further, if $a$ be any number, there are two linearly independent members of $I_{n}{ }^{p+3}$, which contain the factor $(a x)^{p^{p-1}}$ - say $(\alpha x)^{p-1} \psi_{1}(x)$ and $(\alpha x)^{p-1} \psi_{2}(x)$. If, in addition, $\alpha$ be a root of the Jacobian of the $I_{n}{ }^{p-1}$, then the $I_{n}{ }^{p-1}$ contains a third member $(a x)^{p-1} \phi(x)$, of the same form, which will be a linear combination of $(\alpha x)^{p-1} \psi_{1}(x)$ and $(\alpha x)^{p-1} \psi_{2}(x)$. The particular $I_{n}{ }^{p}$ which contains these will be such that it contains two linearly independent members with the factor $(\alpha x)^{p-1}$. It is easy to shew from the determinantal form of the Jacobian of the $I_{n}{ }^{p}$, that it must have $a$ as a double root in this case. ${ }^{1}$ Thus $\alpha$ is a root of the Jacobian of the pencil $\left[J_{p(n-p+1)}\right]$, which thus contains the Jacobian of the $I_{n}{ }^{p-1}$, also as a factor.

Finally, the Jacobians of $I_{n}^{p-1}, I_{n}^{p+1}$ and $\left[J_{p(n-p+1)}\right]$ are of the respective orders:

$$
\begin{aligned}
(p-1)(n+2-p) ;(p+1)(n-p) ; 2 p(n-p+1)-2 \\
=(p+1)(n+2-p)+(p+1)(n-p)
\end{aligned}
$$

thus the Jacobian of the pencil $\left[J_{p(n-p+1)}\right]$ has been completely accounted for.

1This might be seen most simply by taking the two linearly independent members in the form $(\alpha x) p-1 x_{1} \psi_{1}^{\prime}(x),(a x) p-1 x_{2} \psi_{2}(x)$. 
The linear system $I_{n}^{\prime}$ is a single binary form $a_{x}{ }^{n}$, and its Jacobian is also $a_{x}{ }^{n}$. Hence by putting $p=2$ in the above, we have the following theorem:

If $a_{x}{ }^{n}, b_{x}{ }^{n}, c_{x}{ }^{n}$ are linearly independent, and $J_{\lambda}$ denote the Jacobian of $a_{x}{ }^{n}$ with $b_{x}{ }^{n}+\lambda c_{x}{ }^{n}$, then the Jacobian of the pencil $\left[J_{\lambda}\right]$ is the product of $a_{x}{ }^{n}$ with the Jacobian of the net $\left[a_{x}^{n}, b_{x}{ }^{n}, c_{x}{ }^{n}\right]$.

If we now put $n=3$, and let $a_{x}^{\prime}{ }^{3}$ denote the unique cubic apolar to $a_{x}^{3}, b_{x}^{3}, c_{x}^{3}$, then $a_{x}^{\prime}{ }^{3}$ is the Jacobian of the net $\left[a_{x}^{3}, b_{x}{ }^{3}, c_{x}{ }^{3}\right]$; and the above theorem gives a rational determination of the pencil of quartics $\left[J_{\lambda}\right]$, from its Jacobian $a_{x}{ }^{3} a_{x}^{\prime}{ }^{3}$. This proves the original theorem; it is only necessary to satisfy oneself that we do not obtain a different pencil $\left[J_{\lambda}\right]$ by reversing the rôles of the apolar cubics $a_{x}^{3}, a_{x}^{\prime}{ }^{3}$. For this, it is enough to remark that the pencils of cubics which contain $a^{\prime}{ }^{3}$ and are apolar to $a_{x}{ }^{3}$ are merely the apolar pencils of those which contain $a_{v}{ }^{3}$ and are apolar to $a_{x}^{\prime}{ }^{3}$-so that we are led in either case to the same pencil of Jacobians $\left[J_{\lambda}\right]$.

\section{Properties of the Special Pencil.}

III. The pencil of quartics determined rationally in the manner described from a Jacobian which is the product of the apolar cubics $a_{x}{ }^{3}, a_{x}^{\prime}{ }^{3}$, may be conveniently called the special pencil $\left[a_{x}{ }^{3}, a_{x}^{\prime}{ }^{3}\right]$. The fundamental property of the special pencil is given by the theorem:

The two self-apolar members of a special pencil are coalesced; conversely any pencil of quartics with coalesced self-apolar members has a product of two apolar cubics for its Jacobian, and is a special pencil.

The proof is immediate from the fact that among the family of pencils of cubics which contain $a_{x}{ }^{3}$ and are apolar to $a_{x}^{\prime}{ }^{3}$, there is only one which is a Null Pencil-namely the pencil $\lambda_{1} a_{x}{ }^{3}+\lambda_{2} a_{x}{ }^{3}$. Hence among the Jacobians $J_{\lambda}$ there is only one which is self-apolar.

If $a_{x^{\prime}}{ }^{2} \beta_{x}^{\prime} \gamma_{x}^{\prime}$ is a member of the special pencil $\left[\alpha_{x} \beta_{x} \gamma_{x}, a_{x}^{\prime}{ }^{3}\right]$, then $\beta_{x}^{\prime} \gamma^{\prime}{ }_{x}$ is apolar to $\beta_{x} \gamma_{x}$.

This also may be proved from the generation of the special pencil. For if $a_{x} t_{x}{ }^{2}$ is apolar to $a_{x}^{\prime}{ }^{3}$, then the member $a_{x}{ }^{2} \beta_{x}^{\prime} \gamma_{x}^{\prime}$ of the special can only have arisen as the Jacobian of $\alpha_{x} \beta_{x} \gamma_{x}$ and $\alpha_{x} t_{x}{ }^{2}$. Hence $\beta_{u}^{\prime} \gamma^{\prime}{ }_{x}$ is the Jacobian of $\beta_{x} \gamma_{x}$ and $t_{x^{2}}{ }^{2}$ and is therefore apolar to $\beta_{x} \gamma_{x}$. 
These two properties will be recognised as extensions of corresponding properties of the Null Pencil'; one further property of the same kind may also be stated.

The necessary and sufficient condition that the special pencil $\left[a_{i}^{3}, a_{x}^{\prime}\right\rceil$ has a member in common with the special pencil $\left[b_{x^{3}}, b_{x^{\prime}}^{3}\right]$ is that $a_{x^{3}}{ }^{3}, a_{x}^{\prime}{ }^{3}$ be respectively apolar to $b_{x^{3}}, b^{\prime}{ }^{3}$ (or to $b^{\prime} x^{3}, b^{3}$ ).

IV. It will also be of interest to obtain the condition that a given pencil of quartics may be a special pencil, in an invariant form. We shall use for this purpose a method of specifying a general pencil of quartics by means of three quadratics.

The invariant of the third degree, of a binary quartic, usually denoted by $j$, is its catelecticant-that is, its vanishing is the condition that the quartic may possess an apolar quadratic. A pencil $\Gamma$ of quartics must accordingly possess three members $H_{1} H_{2} H_{3}$ with vanishing $j$; the quadratics $\phi_{1} \phi_{2} \phi_{3}$ apolar to $H_{1} H_{2} H_{3}$ may be called the characteristic quadratics of $\Gamma$. It is easy to shew that the characteristic quadratics may be chosen arbitrarily, and serve to specify the pencil unambiguously. It follows from the definition of the characteristic quadratics $\phi_{1} \phi_{2} \phi_{3}$, that the net of quartics apolar to the pencil is the net $\lambda_{1} \phi_{2} \phi_{3}+\lambda_{2} \phi_{3} \phi_{1}+\lambda_{3} \phi_{1} \phi_{2}$.

It is easy to express $H_{1}, H_{2}, H_{3}$ in terms of the characteristic quadratics. If we use the notations:

$$
\begin{gathered}
\left(\phi_{r}, \phi_{s}\right)^{1}=\phi_{r s},\left(\phi_{r}, \phi_{s}\right)^{2}=d_{r s}\left(\phi_{r s}, \phi_{s t}\right)^{2}=D_{t r}(r \neq t), \\
\left(\phi_{r s}, \phi_{r s}\right)^{2}=D_{t t} \quad\{r, s, t=1,2,3\},
\end{gathered}
$$

it follows without difficulty that we can take:

$$
\begin{aligned}
& H_{1}=\frac{\phi_{31}^{2}}{D_{22}}-\frac{\phi_{12}{ }^{2}}{D_{33}} \\
& H_{2}=\frac{\phi_{12}{ }^{2}}{D_{33}}-\frac{\phi_{23}{ }^{2}}{D_{11}} \\
& H_{3}=\frac{\phi_{23}{ }^{2}}{D_{11}}-\frac{\phi_{31}{ }^{2}}{D_{22}} .
\end{aligned}
$$

By taking linear combinations of $H_{1}, H_{2}, H_{3}$, it follows that each member of the pencil whose characteristic quadratics are $\phi_{1} \phi_{2} \phi_{3}$ is of the form

$$
a \frac{\phi_{23}^{2}}{D_{11}}+b \frac{\phi_{31}^{2}}{D_{22}}+c \frac{\phi_{12}^{2}}{D_{33}}
$$

where $a+b+c=0$. The apolar invariant of a quartic of this form

1 Compare Proc. L.M.S. loc. cit. 
is seen to be a constant multiple of $b c d_{11} D_{11}+c a d_{22} D_{22}+a b d_{33} D_{33}$. The two self-apolar members of the pencil are therefore obtained by solving the equations:

$$
\begin{aligned}
b c d_{11} D_{11}+c a d_{22} D_{22}+a b d_{33} D_{33} & =0 \\
a+b+c & =0 .
\end{aligned}
$$

Thus the condition that the pencil with the characteristic quadratics $\phi_{1} \phi_{2} \phi_{3}$ may have coalesced self-apolar members-that is, may be a special pencil, is :

$$
\sqrt{d_{11} D_{11}}+\sqrt{d_{22} D_{22}}+\sqrt{d_{33} D_{33}}=0
$$

\section{Geometrical Applications.}

$V(a)$. The Nodal Cubic.

Let $L_{1} L_{2} L_{3}$ be the points of inflexion, and let any other line cut the nodal cubic in $P_{1} P_{2} P_{3}$. The pencil of points of contact of tangents to the curve from points on the line $P_{1} P_{2} P_{3}$ is a special pencil, whose Jacobian is composed of the two apolar triads $L_{1} L_{2} L_{3}, P_{1} P_{2} P_{3}$.

(b) The Trinodal Quartic.

Since the self-apolar condition is of the second degree, the lines which cut out a self-apolar tetrad on the trinodal quartic must envelope a conic $S$, which must touch the six inflexional tangents. The lines through any point $P$ of the plane cut out a pencil of tetrads on the curve-the two self-apolar members being cut out by the two tangents from $P$ to $S$. Hence if the pencil determined by $P$ is a special pencil, $P$ must lie on $S$. We have therefore the theorem that the points of contact of the six tangents from any point on $S$ to the curve must fall into two mutually apolar triads.

(c) Let $A B C$ be a triangle $I_{0} I_{1} I_{2} I_{3}$ its incentres. The rectangular hyperbolas through $I_{0} I_{1} I_{2} I_{3}$ have $A B C$ for self-polar triangle, and are therefore all inpolar to the circumcircle $S$; further, the circumcircle is also the centre-locus of the pencil of rectangular hyperbolas. Consider the pencil of tetrads $\Gamma$ cut out by the rectangular hyperbolas on $S$. All the rectangular hyperbolas are inpolar, and only one can in addition be outpolar to the circumcircle; hence only one of the rectangular hyperbolas can cut out a self-apolar tetrad. Thus $\Gamma$ 
contains only one self-apolar member, and is therefore a special pencil. It follows easily that the Jacobian of $\Gamma$ is composed of $A B C$ and the three points $p q r$ at which rectangular hyperbolas of the pencil touch the circle $S$. To see this directly, let one of the hyperbolas $H$ cut $S$ in $h_{1} h_{2} h_{3} h_{4}$ and have its centre at $h$ on $S$. Then $h_{1} h_{2} h_{3} h_{4}$ is the Jacobian of the pencil of inscribed triangles of $S$, which are self-polar in regard to $H$; in particular, it is the Jacobian of $A B C$ with the triad $i j h$, where $i, j$ are the circular points. Hence the Jacobian of $\Gamma$ consists of $A B C$ and the triad apolar to $A B C$ and the pair $i j$. Thus $p q r$ is the equilateral triangle apolar to $A B C$-or, what is the same thing, the pedo-parallel equilateral triangle of $A B C .^{1}$

1 (For the theory of pedo-parallelism of inscribed triangles of a circle, see Proc. Camb. Phil. Soc. 23 (1926), p. 253. 